\title{
The effect of target specification on objects fixated during visual search
}

\author{
L. G. WILLIAMS \\ HONEYWELL INC.
}

When a person searches for a target in a cluttered visual field his eye fixations typically fall on objects. The effect of target specification on the probability of fixating different classes of objects was studied. For fields containing objects differing widely in size, color, and shape: a high proportion of searchers' fixations were on objects of a specified color, a moderate proportion of their fixations were on objects of a specified size, and a slight proportion of their fixations were on objects of a specified shape. When two or more target characteristics were specified, fixations were generally based on a single characteristic. It is proposed that the specification of a target creates a perceptual structure which the searcher explores. The study of visual fixations, in effect, is the study of the perceptual structure.

The subject of this paper is the search for targets in cluttered visual fields. The immediate experimental objective was to determine which things an observer fixates when he was provided with specific information about a target. These data will be used eventually to achieve a different goal-to develop a method for relating search time to characteristics of the target and of the background.

Which characteristics of targets are related to search time in cluttered fields? Green, McGill, and Jenkins (1953) found that under some conditions larger targets were located faster than small ones and that upright numbers were located faster than randomly oriented ones. Rhodes (1964) also found that larger targets were found more quickly than small ones. However, Baker, Morris, and Steedman (1960) found target size to be unrelated to search time, although they did find that compact targets were found relatively quickly.

Which characteristics of the background are related to search time? Numerosity is one such characteristic. Green, McGill, and Jenkins (1953), Boynton, Elworth, and Palmer (1958), McGill (1960), Baker, Morris, and Steedman (1960) and Williams and Borow (1963) have found that, with simple geometrical or alphabetical materials, search time is approximately proportional to the number of objects present in the display.

It is not simply the number of objects which is the important quantity, but rather the number of objects which are similar to the target. Green and Anderson (1956) and S. L. Smith (1962) have shown that for fields containing objects of different color, when the target's color is specified, search time is approximately proportional to the number of similarly colored objects in the field. In consonance with these results,
Rhodes (1964) found that search time was negatively correlated with judged distinctiveness of the target's size and shape. S. W. Smith (1961) found search time was greater for fields containing objects more similar to the target in size, shape and contrast.

In sum, the above studies imply that search time depends on a relationship between the target and the background-namely, the similarities to the target of the various objects in the background.

The search act consists of a sequence of fixations which, in cluttered fields, typically fall on objects. It is hypothesized that the probability of a fixation falling on an object of a given class (for example, on objects of a given size or color or shape) is a function of the target specifications for that search task. Also, for a given search task, if the probabilities of fixating objects of each class are known, and if the numbers of objects of each class are known, then the search time distribution can be predicted. Although it is intuitively clear that this can be done, the mathematical details will be presented elsewhere.

The present study is a general attack on the problem of determining where $S$ will fixate as a function of target specification. The specific question being asked was: when the target is specified in terms of its size, color, and/or shape, what proportion of S's fixations are on objects of each size, color, and shape?

\section{Materials}

Each search field contained 100 forms of a given size (about 2.8, 1.9, 1.3, or 0.8 degrees in visual extent), a given color (blue, green, yellow, orange, or pink), and a given shape (circle, semicircle, triangle, square, or cross). A different two-digit number, about 0.3 degrees in height, was printed within each form. The fields were displayed on a 1.22-meter square rear projection screen 1.72 meters in front of the subject. The screen subtended horizontal and vertical visual angles of 39 degrees. A typical field is shown in Fig. 1.

\section{Procedure}

S's task was to locate a target defined by a specific two-digit number. He was also provided with varying amounts of information about the size, color, and shape of the target. Thus, $S$ always knew the target number, either alone or along with one or more other characteristics.

The $35 \mathrm{~mm}$ slides from which the stimuli were projected were arranged in pairs. The first slide of 


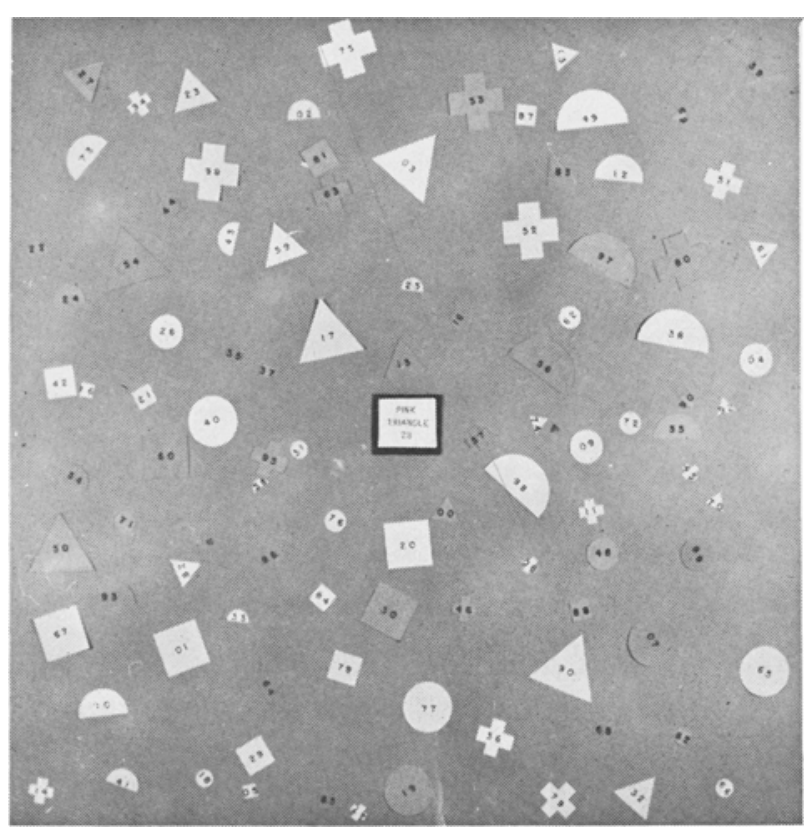

Fig. 1. A search field.

each pair contained the target specification in the form of a two-digit number and a verbal description of the size, color, and/or shape in that order. For the four sizes, the terms very large, large, medium, and small were used. These instructions were then repeated in the center of the second, or search field, slide. Each trial lasted until $\mathrm{S}$ pressed a button indicating that he had found the target. The observer could present as many as 40 consecutive trials to himself by pressing a response button which controlled the slide projector, the automatic camera, and associated apparatus which recorded the eye fixations. There were 200 different search trials per $\mathrm{S}$, with a different search field being used for each trial.

Eye fixations were measured by the corneal reflection technique first used by Dodge and Cline (1901). In the procedure used in our laboratory, S's left eye was largely occluded. An infrared light source produced a virtual image in that eye, which was photographed through a magnification system. The eye fixation record for each trial consisted of a series of time exposures of $4 \mathrm{sec}$. duration.

Each $\mathrm{S}$ required two sessions of 2 to $3 \mathrm{hr}$. duration. Thirty male Ss from colleges and universities in the Minneapolis-St. Paul area took part in the experiment. All Ss possessed normal acuity and color vision as indicated by tests using a Titmus Professional Vision Tester prior to the experiment.

\section{Results}

The raw data consisted of the filmed records of the eye fixations. The records were transformed into tab- ulations of what Ss looked at during each trial by projecting each frame of film (containing about 13 fixations) on the search field, and by using correction procedures to reduce the error. The records from about one-sixth of the trials were not tabulated for two main reasons: the photographs were not in proper focus or the calibration photographs needed for tabulation were unsatisfactory.

Each fixation was tabulated as: (a) falling on a specific object in the field ( 61 percent); (b) at the center of the field containing the information about the target (2 percent); (c) between objects in the field (4 percent); (d) unscored (where the tabulator was not confident enough to classify the fixation into one of the above three categories, 29 percent); (e) a double fixation (when the fixation fell on the object which was just previously fixated, 3 percent). The above percentages are the approximate proportions of fixations within each category.

The accuracy of the eye movement measurement procedure is not a completely determinate quantity, mainly because the ultimate criterion-where the observer was actually looking-is usually not independently known. A more detailed discussion of this question will be presented elsewhere. An indication of the accuracy of the technique used here is provided by the following procedure. When other Ss were instructed to look at specific objects in test fields equal in density to those used in the present study, the data tabulator's "relatively confident" objectidentifications were correct 95 or 98 percent of the time (depending on which of two alignment procedures he used). This should not be taken to suggest that 95 or 98 percent of the objects in Category a, above, were correctly identified, since in the calibration study just discussed all fixations were on objects, whereas in the present study it is presumed that only a fixed proportion were.

\section{Fixation Data}

The approximately 115,000 fixations that could be classified as falling on specific objects (Category a, above) will be the subject of discussion. These data will be treated separately for each type of instruction.

When only the two-digit number was specified, the fixations were unrelated to the color, size or shape of the objects in the field (Table 1, bottom row).

When only the color of the target (in addition to the two-digit number) was specified, there was a strong tendency to fixate objects of that color. When only the size was specified, the tendency to fixate targets of specified size was strong for the largest targets and moderate for the others. When only the shape was specified, the tendency to fixate objects of specified shape was slight.

When the color and size of the target were specified, Ss tended to fixate objects of the specified color just as when color alone was specified. Only when the target was specified to be of the largest size did Ss apparently 
Table 1. Proportion of fixations on objects having the specified characteristics*

\begin{tabular}{|c|c|c|c|c|c|c|c|c|c|c|c|c|c|c|}
\hline \multirow{3}{*}{ Specification } & \multicolumn{14}{|c|}{ Specified Characteristics } \\
\hline & \multicolumn{5}{|c|}{ Color } & \multicolumn{4}{|c|}{ Size (degrees of visual extent) } & \multicolumn{5}{|c|}{ Shape } \\
\hline & B 1 & $\mathrm{Gr}$ & $Y e$ & Or & $\mathrm{Pi}$ & 2.8 & 1.9 & 1.3 & 0.8 & $\mathrm{Ci}_{\mathrm{i}}$ & $\mathrm{Se}$ & $T_{r}$ & $\mathrm{Sq}$ & $\mathrm{Cr}_{\mathrm{r}}$ \\
\hline $\begin{array}{l}\text { Color } \\
\text { Size } \\
\text { Shape }\end{array}$ & .61 & .56 & .59 & .71 & .60 & .59 & .29 & .28 & .35 & 26 & 24 & 24 & 23 & .29 \\
\hline Color + Size & .59 & .65 & .67 & .66 & .59 & .52 & .30 & .30 & .30 & & & & & \\
\hline Color + Shape & .64 & .64 & .66 & .59 & .59 & & & & & .24 & .26 & .27 & .24 & .28 \\
\hline Size + Shape & & & & & & .57 & .30 & .29 & .35 & .27 & .25 & .26 & .24 & .30 \\
\hline Color + Size + Shape & .54 & .55 & .55 & .62 & .54 & .49 & 31 & .29 & .28 & .26 & .28 & .25 & .26 & .26 \\
\hline Number Only & .20 & .20 & .20 & .18 & .22 & .25 & .25 & .26 & .24 & .20 & .20 & .20 & .20 & .20 \\
\hline
\end{tabular}

* To illustrate, when color-plus-size was specified, then for yellow targets 67 percent of the fixations were on yellow objects. When color alone was specified, then for yellow targets 59 percent of the fixations were on yellow objects. The bottom row shows the proportion of fixations on objects of each characteristic indicated by the column label when only the two-digit number was specified.

also use size as a basis for fixation. Although the proportions in Table 1 suggest that Ss also used other sizes as a basis for fixating objects, it is likely that those proportions are increased as a result of finding the target on the last fixation. To show this, assume that Ss used only color and completely ignored size. The number of fixated objects required to find these targets is usually small-a mean of about 18 objects. If let us say, 18 objects are fixated, and the first 17 objects are selected independently of size, then the expected number of specified size would, therefore, be $17 \times \frac{24}{99}+1=5.12$. The proportion $5.12 / 18=.28$ is of comparable value to those in the table for sizes other than the largest.

When the color and shape were specified, there was a strong tendency to fixate objects of specified color, and little or none (considering the argument in the last paragraph) to fixate objects of specified shape. When size and shape were specified, Ss fixated objects on the basis of both size and shape.

When color, size, and shape were specified, fixations were only related to color, the other information being ignored except when the target was also specified to be very large. The overall proportion of fixation on objects of the specified color is less here than for the colorplus-size condition. The statistical significance of this difference is high $\left(X^{2}=96.7\right.$ with $\mathrm{df}=1$ ).

Another question of interest involves what $S$ looks at when he does not look at objects having the specified characteristics. With the color specification there is no systematic tendency to look at any other color. However,

Table 2. Proportion of fixations on objects of different size for each size specification

\begin{tabular}{lcccc} 
& \multicolumn{4}{c}{ Size of Fixated Object } \\
Specified Size & Very Large & Large & Medium & Small \\
\cline { 2 - 5 } Very Large & 0.59 & 0.24 & 0.11 & 0.07 \\
Large & 0.35 & 0.29 & 0.21 & 0.15 \\
Medium & 0.23 & 0.26 & 0.28 & 0.22 \\
Small & 0.13 & 0.20 & 0.31 & 0.35 \\
\hline
\end{tabular}

with the size specifications, Table 2 shows that the likelihood of fixating an object depends on its similarity to the specified size. For three sizes, observers are most likely to fixate objects of specified size. For the "Large" specification the peak has shifted to the largest size. This may be a result of the specific words used in the instructions.

\section{Search Times}

The mean times required to find targets for the eight different specifications are shown in Table 3. As can be seen in the table, the specifications divide themselves into four classes: color specified, size but not color specified, shape but not color or size specified, and number only specified. The search times are increasingly greater for the four classes. These data correspond directly to the efficiency of looking as measured by the proportion of fixations on objects having the specified characteristics as shown in Table 1.

\section{Discussion}

The results can be summarized as follows: for a field containing objects differing widely in size, color, and shape, Ss selectively fixated objects much better on the basis of color than on size or shape. Further, when provided with information about two or three target characteristics, Ss generally fixated objects on the basis of a single characteristic, namely color if provided.

How should these data be interpreted? The search act consists of two main components-identification and acquisition. Identification is the classification of the foveally imaged object-this object is or is not the target. Acquisition is the selection of an objector point outside of the fovea to look at next. S's ability to selectively fixate objects on the basis of specific characteristics is, in fact, his ability to select objects in the extra-foveal field which are similar to the target as specified.

There are two alternative hypotheses about the process by which objects are acquired. One hypothesis is 
Table 3. Mean time to find target for different specifications

\begin{tabular}{lcc} 
Specification & Mean Time (seconds) & Number of Trials \\
\hline Color & 7.6 & 455 \\
Color and Size & 6.1 & 463 \\
Color and Shape & 7.1 & 571 \\
Color and Size and Shape & 6.4 & 1178 \\
Size & 16.4 & 468 \\
Size and Shape & 15.8 & 457 \\
Shape & 20.7 & 461 \\
Number Only & 22.8 & 579
\end{tabular}

that the selection of each new object to fixate is a choice reaction task for the $\mathrm{S}$. At any moment in time he is likely to be looking at a given object or point in the field. After having decided that thatobject is not the target, the next object is selected from the many visible ones in the extra-foveal field. The objects in the field can be seen with diminishing clarity with increasing distance from the fixation point. The hypothesis is that $S$ makes a choice of one such object on the basis of the target specifications in at most about $300 \mathrm{msec}$. (since he typically makes more than three fixations per second).

The other hypothesis is that S's percept of the total field is determined by the target specifications. When, for example, he searches for an orange target he perceives a patterning of orange objects on a background of other colored objects. He tries to look at different parts of this pattern until he comes to the target. Thus, when $S$ searches for an orange square the figural structure consists of orange objects rather than orange square objects, simply because he is incapable of structuring the field in terms of both characteristics. S's ability to selectively fixate objects on the basis of any specific set of characteristics is determined by how well he can perceptually structure the field in terms of those characteristics. The data presented would suggest, for example, that in a field containing the five shapes used here, shape is a poor basis for such structure.

At this time the data do not conclusively support one of the hypotheses to the exclusion of the other. The author tentatively accepts the perceptual structuring hypothesis since the alternative has a major weakness with respect to the time constraints. Although up to $300 \mathrm{msec}$. may be available for the choice reaction task, it is likely that only a fraction of this interval can actually be used since time is required for object identification and for eye movements. Since the simple reaction time for visual stimuli is about $180 \mathrm{msec}$. (Woodworth \& Schlosberg, 1960), it appears that there may be insufficient time for the hypothesized complex choice reaction.

\section{References}

Baker, C, A., Morris, D. F., \& Steedman, W. C. Target recognition on complex displays. Hum. Factors, 1960, 2, 51 .

Boynton, R. M., Elworth, C., \& Palmer, R. M. Laboratory studies pertaining to visual air reconnaissance. WADC Tech. Rep. 55304, Part IIl, Wright-Patterson Air Force Base, Ohio, April 1958.

Dodge, R., \& Cline, T. S. The angle velocity of eye movements. Psychol. Rev., 1901, 3, 145 .

Green, B. F., \& Anderson, L. K. Color coding in a visual search task. J. exp. Psychol., 1956, 51, 19.

Green, B. F., McGill, W. J., \& Jenkins, A. M. The time required to search for numbers on large visual displays. MIT, Lincoln Laboratory, TR36, 1953.

McGill, W. J. Search distributions in magnified time. Visual search techniques. Washington: National Academy of Science - Nat. Res. Coun., 1960 (Pub. 712).

Rhodes, F. Predicting the difficulty of locating targets from judgments of image characteristics. Technical Documentary Report AMRL-TDR-64-19, Aerospace Medical Research Laboratories, Wright-Patterson Air Force Base, Ohio, March 1964.

Smith, S. L. Color coding and visual search. J. exp. Psychol., $1962,64,434$.

Smith, S. W. Time required for target detection in complex abstract visual display. Memorandum of Project Michigan 2900-235-R, Institute of Science and Technology, The University of Michigan, April 1961.

Williams, L. G., \& Borow, Marion. The effect of rate and direction of display movement on visual search. Hum. Factors, 1963, 5, 139.

Woodworth, R. S., \& Schlosberg, H. Experimental psychology, New York: Henry Holt and Company, 1960.

\section{Note}

1. This work has been supported by the Engineering Psychology Branch, Office of Naval Research, under Contract NONR 4774(00).

(Accepted for publication July 28, 1966.) 